Original article

\title{
Quantification of heat threshold and tolerance to evaluate small fiber neuropathy- an indigenously developed thermal model of pain
}

\author{
P. Rama Mohan ${ }^{a}$, Swetha chowdary ${ }^{b}$, A.V. Siva Kumar ${ }^{\text {c, }}$, K.N. Maruthy ${ }^{c}$, K. Mahesh kumar ${ }^{\mathrm{d}}$ \\ ${ }^{a}$ Dept. of Pharmacology, Narayana Medical College, Nellore, Andhra Pradesh, India \\ ${ }^{\mathrm{b}}$ Narayana College of Physiotherapy, Nellore, Andhra Pradesh, India \\ ${ }^{\mathrm{c}}$ Dept. of Physiology, Narayana Medical College and Hospital, Nellore, Andhra Pradesh, India \\ d Dept. of Physiology \& Biochemistry, Govt. Yoga and Naturopathy College \& Hospital, Chennai, Tamilnadu , 600104, India
}

\section{A R T I C L E I N F O}

\section{Keywords:}

Minimum heat threshold

Maximum heat tolerance

Small fiber neuropathy

Thermal model of pain

\begin{abstract}
A B S T R A C T
Introduction: A wide variety of diseases alter the perceptions of different sensations, often evaluated in a subjective manner. Assessment of temperature perception and tolerance is a useful screening tool to evaluate the Degenerative and neuropathic changes of an individual. Therefore the current study was intended to design and develop an inexpensive device to quantify the heat threshold and tolerance in healthy participants.

Materials and methods: The study was carried out in 30 apparent healthy participants for heat threshold, and tolerance was recorded on both hands' thenar and dorsal sites on two occasions. The minimum temperature when the subject was perceived is threshold and maximum until the subject withstood tolerance. The data was collected using the electronically controlled device for these two extremes. The entire study was carried out at a controlled room temperature precisely.

Results: The heat threshold was $39.84 \pm 2.33^{\circ} \mathrm{C}$, and the tolerance was perceived at $46.84 \pm 3.36{ }^{\circ} \mathrm{C}$. There were no intraindividual differences $(p>0.05)$ in the heat threshold measured on two different periods as well as between the two hands $(p>0.05)$. As expected, there were significantly higher threshold values on the palm's thenar aspect than dorsum $(p<0.05)$. The tolerance was significantly higher in the thenar aspect than the dorsum of both hands. ( $p<0.01, p-0.03$ ). There were no significant inter-hand differences of both surfaces of the hand.

Conclusion: Our study showed that the results of threshold and tolerance using the indigenously built device were consistent and reproducible proves the robustness of the methodology. It is a cost-effective and user-friendly device that provides quantitative results of temperature extremes.
\end{abstract}

\section{Introduction}

The peripheral nerve fibers convey various sensations from peripheral organs to cortical sensory areas. These fibers are classified into A, B $\& \mathrm{C}$ fibers based on their diameter, myelination, and conduction velocity. C fibers are responsible for controlling autonomic balance, slow pain \& temperature conduction. ${ }^{1}$ They are unmyelinated, less in conduction velocity $(<2 \mathrm{~m} / \mathrm{s})$. The degenerative changes in these fibers due to various reasons cause small fiber neuropathy. ${ }^{2,3}$ The primary etiological elements for small fiber neuropathy are Diabetes, hypothyroidism, endocrine disorders, hereditary diseases, and other potentially infectious diseases. ${ }^{4}$ The symptoms of small fiber neuropathy are highly variable includes burning, tingling, prickling, and the common is short bursts of pain5. These symptoms often extend in limbs as "stocking-glove distribution." It also cultivates autonomic derangement, which primes disturbance in visceral organs. ${ }^{6}$

Small fiber neuropathy often diagnosed using various electrophysiological procedures like nerve conduction studies, electromyography (EMG) in addition to imaging techniques. However, these fibers having small diameters and cannot implement these tests to evaluate functional impairment. ${ }^{7}$ The classic test to assess the small fiber injury is temperature and pain perception. Quantification of experimental pain depends on the type of noxious stimuli used, their application, and the assessment method where the stimuli are often applied in fixed or ascending

\footnotetext{
* Corresponding author. Department Of Physiology, Narayana Medical College, Nellore, 524003, India.

E-mail addresses: prammohan@narayanamedicalcollege.com (P.R. Mohan), swethachowdarykamineni17@gmail.com (S. chowdary), asivakumar@ narayanamedicalcollege.com (A.V. Siva Kumar), dr.maruthy@gmail.com (K.N. Maruthy), doctor.mahesh1985@gmail.com (K. Mahesh kumar).
} 


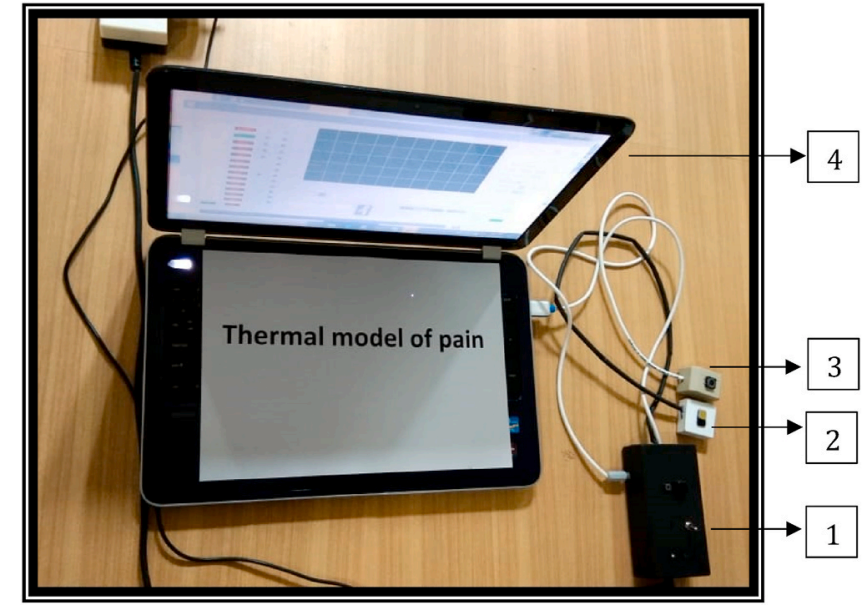

Fig. 1. Thermal model of Pain Device with components

1) temperature organizing box, 2) heat-producing metal plate (LM-35 thermal sensor), 3) temperature control button for participant safety, 4) PC for real time thermometry with oscilloscope (DAQ smart).

magnitudes. ${ }^{8}$ They can be assessed using threshold and tolerance measurements and subjectively characterized by pain scores. ${ }^{9}$ The temperature perception has two extremes, minimal heat threshold (MHT) and maximum temperature tolerance (MTT). Hence these quantitative objective variables of temperature perception provide potential information about small fiber function. ${ }^{10,11}$ We designed and developed a household device to quantify temperature threshold and tolerance, reflecting small fiber activity. Therefore, we focused on the psychophysics of heat stimuli when applied to the thenar and dorsum of the hand and digitized the data of two extremes of perception and tolerance. Thus, The present study aimed to characterize the device and established methodology for MHT and MTT in healthy participants.

\section{Materials \& methods}

\subsection{Instrument design}

The computer-based electronically controlled heat-producing device is an in-house built tool using a USB DAQ -Oscilloscope for data acquisition. It has four components $\mathrm{A}$ ) temperature organizing box, B) heat-producing metal plate, C) temperature control button for participant safety, and D) PC for real time thermometry. The temperature sensor used in the system was a fast response pre-calibrated IC-LM35 with a response time of less than $2 \mathrm{~s}$. The heating device was a solid-state semiconductor-based heater with a cross-sectional area of 1 sq. $\mathrm{cm}$ and is controlled electronically. The data was displayed on the oscilloscope of the DAQ application as shown in figure - 1 .

\subsection{Study population}

The study protocol was reviewed and approved by the Institutional ethic committee, Narayana Medical College and Hospital, Nellore. The study was carried out in the 30 participants who are medical students and other technical staff of the hospital premises acquainted with the investigator using non - random sampling technique (Convenient sampling). Apparent healthy participants aged between 18 and 25 years were recruited into the current study. The subjects with a history of known acute or chronic medical illness, chronic smokers, alcoholics, and intake of centrally-acting drug patients were excluded from the study. The entire procedure was explained to each participant and their right to withdraw from the study at any time. According to the Declaration of Helsinki (1975) and later amendments, written informed consent was obtained from all participants. There were 13 males and 17 females
Table 1

Shows heat threshold and Tolerance.

\begin{tabular}{llcr}
\hline Threshold & Mean & & 39.84 \\
& 95\% Confidence Interval for Mean & Lower & 39.32 \\
& Median & Upper & 40.36 \\
& & 39.90 \\
Std. Deviation & & 2.33 \\
Minimum & & 34.7 \\
Maximum & & 46.2 \\
& Range & 11.5 \\
\hline & & \\
\hline Tolerance & Mean & & 46.84 \\
& 95\% Confidence Interval for Mean & 46.09 \\
& Lower & 47.59 \\
& Median & Upper & 46.40 \\
& & 3.36 \\
& Std. Deviation & 40.60 \\
Minimum & & 58.60 \\
Maximum & & 18.00 \\
\hline Range & &
\end{tabular}

among the participants recruited for the study. The room temperature at which this protocol was executed was $32-35{ }^{\circ} \mathrm{C}$ to avoid confounding results on the outcome. The data acquisition was executed in the participants to record threshold and tolerance after explaining the procedure and demonstrated one trial to each participant. Later, the two extremes of temperature perception were measured two times in the thenar and dorsum aspect of the hand and two successive days to find out the reproducibility.

\subsection{Outcome measurement}

The outcome was measured in terms of two extremes of temperature.

\subsubsection{Minimum heat threshold (MHT)}

The minimum temperature at which the subject is perceived the warmth in the device. The dorsum of the hand is placed over the heated metal, and the device was heated up until the subject is felt the warmth. The subject also receives a safety control button to let us know the perception point of temperature. The same procedure is repeated on another side (thenar aspect) of the hand, and twice the data is recorded at each location with an interval of $20 \mathrm{~min}$.

\subsubsection{Maximum temperature tolerance (MTT)}

It is the maximum temperature at which the subject cannot tolerate further heat. The subject can press the standard control button to let us know the threshold and tolerance recorded on the computer screen. This procedure was also repeated on both sides as well as in both hands of each participant.

\subsection{Statistical analysis}

The data analysis was carried out using ' $R$ ' software. The data sets were represented as Mean $\pm \mathrm{SD}$. The normality of data was tested using the Kolmogorov-Smirnov test. Descriptive statistics were analyzed and expressed for each variable of temperature. As the data sets were skewed from a normal distribution, non-parametric tests were implemented to determine differences between the thenar and dorsum of the hand.

\section{Results}

The mean age of the participants was $18 \pm 1.5$ years. The mean threshold temperature (MHT) was $39.84 \pm 2.33^{\circ} \mathrm{C}$, whereas the tolerance was experienced at $46.84 \pm 3.36{ }^{\circ} \mathrm{C}$, as shown in Table - 1 . There were no intraindividual differences $(p>0.05)$ in pain threshold as measured on two different periods. Similarly, the threshold values were statistically similar between the two hands $(p>0.05)$. As expected, there 
Table 2

Effect of Period, dexterity and site on heat Threshold (MHT).

\begin{tabular}{|c|c|c|c|c|c|c|c|c|}
\hline Recordings & Dexterity & Site & Mean & SD & Median & Min & Max & $p$-value \\
\hline \multirow[t]{4}{*}{ Primary } & Left & Dorsum & 39.2 & 2.4 & 39.6 & 34.7 & 43.5 & $p<0.05$ \\
\hline & Left & Thenar & 40.8 & 1.9 & 41.1 & 37.6 & 44 & \\
\hline & Right & Dorsum & 39 & 2 & 39.1 & 35.7 & 42 & $p>0.05$ \\
\hline & Right & Thenar & 40.4 & 2.6 & 41.1 & 36.1 & 46.2 & \\
\hline \multirow[t]{4}{*}{ Secondary } & Left & Dorsum & 39.8 & 2.1 & 40.6 & 36.2 & 42.5 & $p<0.05$ \\
\hline & Left & Thenar & 41.1 & 1.8 & 41.5 & 36.2 & 43.5 & \\
\hline & Right & Dorsum & 39.8 & 2.1 & 40.6 & 35.2 & 42.5 & $p>0.05$ \\
\hline & Right & Thenar & 40.7 & 2.6 & 40.9 & 36.1 & 46.4 & \\
\hline
\end{tabular}

Table 3

Effect dexterity and site on heat tolerance (MTT).

\begin{tabular}{llllllll}
\hline \multirow{2}{*}{ Dexterity } & Site & Mean & SD & Median & Min & Max & $p$-value \\
\hline \multirow{2}{*}{ Left } & Dorsum & 45.54 & 2.27 & 45.05 & 42.5 & 49.3 & $p<0.01$ \\
& Thenar & 48.29 & 3.78 & 47.40 & 43.9 & 58.6 & \\
\multirow{2}{*}{ Right } & Dorsum & 45.45 & 2.53 & 45.10 & 40.6 & 50.5 & $p-0.03$ \\
& Thenar & 48.12 & 3.67 & 47.65 & 43.5 & 53.5 & \\
\hline
\end{tabular}

All the numerical values are expressed in Celsius.

were significantly higher threshold values on the thenar aspect of the palm than dorsum $(p<0.05)$, as shown in Table -2 . The tolerance of temperature (MTT) was significantly higher in the thenar aspect of the palm than the dorsum surface of both hands ( $p<0.01$, p-0.03), as displayed in Table -3 . However, there were no significant differences between both hands ( $p>0.05$ - Results were not displayed).

\section{Discussion}

The current study was intended to evaluate the characterization and robustness of an indigenously developed $\&$ in-house built device for heat threshold and heat tolerance in healthy participants.

The non invasive and quantitative assessment of any variable would be more informative inorder to screen and diagnose subclinical conditions. There is enormous transition in the electronics and it has been even encroached in medicine for various purposes. The authors of current study has been working on inexpensive and non invasive evaluation of autonomic neuropathy using heart rate variability, dynamic pupillometry. ${ }^{12,16}$ In addition we are also working on utility of sensory perception interms of heat threshold and tolerance provide us the broad range of peripheral nerve conduction especially small fiber activity.

The lab - based assessment of individual changes in pain sensitivity can predict differences in acute, chronic pain, and prognosis. Jensen TS et al. (1991) concluded that the clinical applications of their findings are broad and quite important. However, performing sophisticated quantitative sensory testing requires time, expertise, and equipment not readily available in many clinical settings. It would be helpful to know whether simply enquiring participants about their pain sensitivity provides information similar to that obtained from experimental pain testing. ${ }^{13}$ The experimental and clinical studies on acute pain have shown that women are more sensitive to pain. Schaffner N et al. (2008) showed that men have a higher pain tolerance than women for induced heat pain. The still reduced number of subjects prevent from computing normative estimates. Nevertheless, in assessing the basic psychophysical properties of pain threshold and tolerance, their data may contribute to a more conclusive interpretation of brain imaging studies aiming to assess laterality effects. ${ }^{14}$ However in our study, we did not concentrate on gender discrimination in perception of heat threshold and tolerance. But we found there was a significant change in both surfaces of hand due to thickness of the skins. It can be attributed to anatomical variation temperature perception. But there is difference wa found out between two hands indicate symmetrical sensory perception. However, some studies have postulated cerebral dominance can discriminate the threshold and tolerance of heat. ${ }^{15} \mathrm{~A}$ familiar feature in diabetic neuropathies is emerging painless ulcers, which remain unnoticed in quite a few patients. We, therefore, introduced a warm-sensibility index in which heat is perceived but not reported as painful. ${ }^{17}$ We believe that this measure may be a screening tool in witnessing deterioration of small nerve fiber service in neuropathies. ${ }^{18}$ Further studies are necessary to establish the reproducibility and validity of this measure. However, we would note that the reproducibility of the thermal threshold measures has been demonstrated in several previous studies as mentioned earlier. There is paucity in literature and few studies were performed int his domain. Revealing of early and subclinical neuropathy is an important task in the treatment and handling of diabetic patients. The neuropathies are clinically and electrophysiological heterogeneous, and there is no simple relationship between peripheral nerve function and glycemic control.

\section{Limitations}

The present study has some limitations. This study was only established in healthy volunteers who are acquainted with the investigator. The results can not generalize and the study was intended to prove te concept rather to provide results. The sample size was less, and relationships with other dependent variables were not established. This protocol will be extrapolated in different neuropathic patients to characterize the study results for accuracy and reliability.

\section{Future steps}

The device utility will revealed by extending the study in multiple suspected population and implementing appropriate study designs. The robustness of methodology and execution will be further unvield during data collection. The data analysis will be done by developing the platform to produce automated results.

\section{Conclusion}

The current study reports suggest consistent and reproducible values in heat threshold and heat tolerance to the thermal model of pain with little variations. It is cost-effective, a portable, user-friendly device that provides reliable readings to evaluate small fiber neuropathy and response to treatment quantitatively.

\section{Ethical considerations}

The study design was reviewed and approved by the host institution's Institutional Ethics committee.

\section{Funding}

There is no financial support for this research work and publication of the article.

\section{Declaration of competing interest}

On behalf of all the authors, the corresponding author states no 
potential conflict of interest regarding the research work and publication.

\section{Acknowledgment}

We would like to express my sincere gratitude to Mr. Vivek, Mavom lab, PVT. Ltd, Bangalore, for helping in the design of the instrument. The authors would like to express their gratitude to Narayana Medical College's management for their constant support and encouragement throughout study period.

\section{References}

1 Manzano GM, Giuliano LM, Nóbrega JA. A brief historical note on the classification of nerve fibers. Arquivos de neuro-psiquiatria. 2008;66(1):117-119.

2 Lacomis D. Small-fiber neuropathy. Muscle \& Nerve. Official Journal of the American Association of Electrodiagnostic Medicine. 2002;26(2):173-188.

3 Low VA, Sandroni P, Fealey RD, Low PA. Detection of small-fiber neuropathy by sudomotor testing. Muscle Nerve: Official Journal of the American Association of Electrodiagnostic Medicine. 2006;34(1):57-61.

4 Tavee J, Zhou L. Small fiber neuropathy: a burning problem. Cleve Clin J Med. 2009; 76(5):297-305.

5 Hovaguimian A, Gibbons CH. Diagnosis and treatment of pain in small-fiber neuropathy. Curr Pain Headache Rep. 2011;15(3):193-200.

6 Smith T, Trojaborg W. Somatosensory evoked potentials in the evaluation of patients with stocking/glove paresthesias. Acta Neurol Scand. 1989;79(1):63-67.

7 Lauria G, Hsieh ST, Johansson O, et al. European federation of neurological societies peripheral nerve society guideline on the use of skin biopsy in the diagnosis of small fiber neuropathy. Report of a joint task force of the European federation of neurological societies and the peripheral nerve society. Eur J Neurol. 2010;17(7), e49, 903.
8 Tobin K, Giuliani MJ, Lacomis D. Comparison of different modalities for detection of small fiber neuropathy. Clin Neurophysiol. 1999;110(11):1909-1912.

9 Treede RD, Meyer RA, Raja SN, Campbell JN. Evidence for two different heat transduction mechanisms in nociceptive primary afferents innervating monkey skin. J Physiol. 1995;483(3):747-758.

10 Dyck PJ, Zimmerman I, Gillen DA, Johnson D, Karnes JL, O’Brien PC. Cool, warm, and heat-pain detection thresholds: testing methods and inferences about anatomic distribution of receptors. Neurology. 1993;43(8):1500.

11 MaheshKumar K, Maruthy KN, Padmavathi R. Comparision of photo pulse plethysmography module with Mobil-O-graph for measurement of pulse wave velocity. Clinical Epidemiology and Global Health. 2021;9:216-220.

12 Maruthy KN, Padmavathi R, Sowjanya B, MaheshKumar K. Quantitative determination of pupil by dynamic pupillometry using infrared videography-Role in evaluation of autonomic activity. Clinical Epidemiology and Global Health. 2020;8(3): 728-732.

13 Jensen TS, Bach FW, Kastrup J, Deigaard A, Brennum J. Vibratory and thermal thresholds in diabetics with and without clinical neuropathy. Acta Neurol Scand. 1991;84(4):326-333.

14 Schaffner N, Wittwer A, Kut E, Folkers G, Benninger DH, Candia V. Heat pain threshold and tolerance show no left-right perceptual differences at complementary sites of the human forearm. Neurosci Lett. 2008;440(3):309-313.

15 Breivik H, Borchgrevink PC, Allen SM, et al. Assessment of pain. Br J Addiction: Br J Anaesth. 2008;101(1):17-24.

16 Pullaganti M, Kumar SA, Maruthy KN, Gurja JP, Chintala KK. Association of stress with heart rate variability in different phases of the menstrual cycle. Natl J Physiol Pharm Pharmacol. 2019;9(3):256-260.

17 Sivakumar AV, Kalburgi-Narayana M, Kuppusamy M, Ramaswamy P, Bachali S. Computerized dynamic pupillometry as a screening tool for evaluation of autonomic activity. Neurophysiol Clin. 2020;50(5):321-329.

18 Kumar CK, Maruthy KN, Sasikala P, Gurja JP, Kumar AV, Kareem SK. Impact of chronic alcoholism on temporal cognition and coordination of motor activity. International Journal of Physiology. 2018;6(4):124-127. 